\title{
Activités
}

1-1 | avril 2004

Varia

\section{Connaissances et interventions}

\section{Charles Gadbois et Jacques Leplat}

\section{OpenEdition}

Journals

Édition électronique

URL : http://journals.openedition.org/activites/1138

DOI : 10.4000/activites. 1138

ISSN : 1765-2723

\section{Éditeur}

ARPACT - Association Recherches et Pratiques sur les ACTivités

\section{Référence électronique}

Charles Gadbois et Jacques Leplat, « Connaissances et interventions », Activités [En ligne], 1-1 | avri 2004, mis en ligne le 02 avril 2004, consulté le 01 mai 2019. URL : http://journals.openedition.org/ activites/1138; DOI : 10.4000/activites.1138

\section{(2) $\odot \Theta \Theta$}

Activités est mis à disposition selon les termes de la licence Creative Commons Attribution - Pas d'Utilisation Commerciale - Pas de Modification 4.0 International 


\title{
Connaissances et interventions
}

\author{
Charles Gadbois \\ Directeur de recherches CNRS, \\ Laboratoire d'Ergonomie Physiologique et cognitive, \\ 41, Rue Gay-Lussac, 75005 Paris \\ Charles.Gadbois@wanadoo.fr \\ Jacques Leplat \\ Directeur honoraire à l'Ecole Pratique des Hautes Etudes, \\ 41, Rue Gay-Lussac, 75005 Paris, \\ Jacques.Leplat@wanadoo.fr
}

RÉSUMÉ

Cet article s'inscrit dans le cadre des débats récurrents en ergonomie sur les rapports de la recherche et de la pratique. Il se propose de montrer que ces débats peuvent être générateurs de progrès dans les différents champs de connaissances de la discipline. Dans une première partie sont distinguées les différentes catégories de connaissances ergonomiques, leurs origines et leurs places dans les interventions ergonomiques. La seconde partie met en lumière les connaissances que tout professionnel acquiert à travers son expérience personnelle. La troisième analyse les conditions de la production et de la capitalisation de connaissances nouvelles au cours des interventions. La conclusion dégage quelques pistes de réflexion et suggère des voies d'action susceptibles d'aider à surmonter les difficultés présentes pour de meilleures coopérations entre les différents acteurs de l'ergonomie.

\section{MOTS-CLÉ}

Connaissances, intervention, interactions recherche-pratique

\begin{abstract}
This paper echoes the recurrent debates on the relationship between research and practice in ergonomics. It proposes to show that these debates can enhance progress in the various fields of the discipline. Firstly, the various categories of ergonomic knowlege are characterised according to their origins and their contribution to field operations. Secondly, light is shed on knowledge acquired by each practitioner through his personal experience. Thirdly the producing conditions of new knowledge in the course of ergonomic operations and the means of their capitalisation are examined. The conclusion draws some guidelines for further reflections and suggests several ways to overcome the present difficulties and work towards a better cooperation between the various actors of ergonomics
\end{abstract}

KEYWORDS

Knowledge, transformation action, research-practice relationship 
En arrière-fond de ce texte, on aura vite fait de reconnaître les échos d'un débat qui hante les couloirs de la SELF, débat concernant les rapports de la recherche et de la pratique, des chercheurs et des praticiens. Les auteurs sont ou ont été des chercheurs par leur place dans les institutions auxquelles ils appartiennent, ce qui ne les a pas empêchés d'avoir toujours eu des liens avec des collègues engagés dans la pratique. Aussi regrettent-ils les tensions qui existent entre ces deux pôles de l'ergonomie. Le présent texte n'a pas la prétention de faire disparaître ces tensions aux composantes multiples, mais d'en éclairer des sources avec quelques distinctions utiles. Pour cela, nous réfléchirons à la question suivante : qu'appelle-t-on connaissances en ergonomie et quels sont les rapports de ces connaissances avec les pratiques ergonomiques ? Certains pourront juger présomptueux de parler de pratiques ergonomiques sans en avoir une expérience directe, en tant que professionnels. Mais, outre que nous n'avons pas la prétention de donner de leçon à quiconque, notre but est d'essayer de baliser un champ complexe pour éviter des discussions ambiguës. Il y a certes à débattre sur les problèmes qui viennent d'être évoqués, mais qu'au moins, ce débat se fasse sur de bonnes bases et contribue au développement de l'ergonomie sous toutes ses formes : c'est à quoi nous voudrions contribuer par ce texte.

Dans une première partie nous nous attacherons à préciser les différentes catégories de connaissances et leur place dans les interventions ergonomiques. Nous tenterons ensuite de mettre en lumière la part des connaissances particulières que tout professionnel acquiert à travers son expérience personnelle. Dans un troisième temps, nous esquisserons des pistes de réflexion sur les conditions de la production et de la capitalisation de connaissances nouvelles par les interventions.

\section{1.- Gamme et diversité des connaissances ergonomiques}

Le savoir ergonomique est à la fois dans des documents écrits et dans la tête des ergonomes. Les compétences acquises par l'expérience ne sont pas l'image des connaissances qui figurent dans les documents, même si elles les recoupent partiellement. Ces compétences représentent un savoir opératoire, fonctionnel qui s'inscrit dans les actions des intervenants, mais qui n'est pas facilement communicable, ni verbalisable. Dans cette première partie, on s'intéressera au premier type de savoir, celui qui est extériorisé, qui est transmissible et exploité dans la formation, mais aussi dans la pratique pour compléter les acquis antérieurs et communiquer des acquis nouveaux.

\section{1.- Supports des connaissances ergonomiques}

Les connaissances ergonomiques figurent dans des documents de types divers :

- des livres à thème général (traités, manuels, guides,...) ou plus spécialisés, comme beaucoup de livres des collections Octarès, Le Travail Humain, ANACT, Taylor \& Francis, etc. ;

- des articles de revue : Le Travail Humain, Performances (aujourd'hui disparu, mais non absent des bibliothèques), Ergonomics, Applied Ergonomics, Education Permanente, etc. ;

- des Actes divers issus de congrès, colloques, journées, séminaires ;

- des rapports dont le nombre s'est multiplié avec celui des ordinateurs ;

- des sites Internet (PISTES, INRS, ANACT, mais aussi bien d'autres ...).

Ces différents types de documents sont répertoriés, surtout ceux du début, dans des banques de données et dans les Ergonomics Abstracts. Mais pour celui qui est loin des grandes bibliothèques, l'accès à ces documents n'est pas facile. Il y aurait sans doute profit à s'interroger sur les moyens de surmonter ces difficultés par un meilleur développement de l'utilisation du système Internet. Ces documents font l'objet de codage en vue de classement : on en trouve des exemples dans Ergonomics Abstracts et dans Human Factors. Nous voudrions ici présenter un autre type de classement des connaissances, plus directement en rapport avec les usages ergonomiques. Les catégories propo- 
sées ne sont pas toujours parfaitement disjointes et certains cas semblent difficilement classables dans une seule catégorie. Ceci n'est pas essentiel car cette difficulté aide à cerner les caractéristiques du cas en question.

\section{2.- Connaissances ergonomiques centrées sur des types de situation}

On rangerait ici les connaissances typiquement ergonomiques issues de l'analyse, de la conception ou de l'aménagement de situations de travail. Ces situations sont considérées dans leur complexité, de points de vue divers que l'ergonome essaie de coordonner et dont il essaie aussi de hiérarchiser l'importance par rapport au but et aux conditions de l'étude ou de l'intervention. Les connaissances de cette catégorie sont souvent synthétisées dans des chapitres d'ouvrage. Voici quelques titres de textes illustrant cette catégorie : L'ergonomie de la conduite de projet, Conception d'espaces de travail, Cartes et aides à la navigation, Aménagement de la conduite de ponts roulants.

\section{3.- Connaissances centrées sur les milieux de travail}

Les problèmes ergonomiques portent souvent la marque du milieu dans lequel ils apparaissent (industries, services, transports,...). On peut l'expliquer par le fait que ces problèmes (contraintes physiques, exigences cognitives, pression temporelle, horaires postés, ...) ont des dimensions communes pour un même milieu, un même contexte. Il sera donc souvent très instructif de regrouper des études d'un même domaine, d'en dégager un tableau d'ensemble susceptible de suggérer des interventions plus coordonnées et de faire émerger des solutions de portée plus générale. Ainsi, dans le futur Traité d'Ergonomie dirigé par Falzon (à paraître), trouve-t-on des chapitres sur l'ergonomie hospitalière, sur la conduite automobile ou le secteur du BTP.

\section{4.- Champs de connaissances impliqués par l'ergonomie}

On classerait ici les recherches conduites dans un cadre disciplinaire, mais suscitées par des problèmes ergonomiques ou susceptibles d'être directement exploitées par des études ergonomiques. Avant d'en préciser le statut, on donnera quelques exemples pour trois types de disciplines :

- En rapport avec la psychologie : Exploration visuelle dans la conduite automobile; Mécanismes du travail en temps partagé ; Analyse des activités cognitives en situation dynamique ; Conception des documents prescriptifs.

- En rapport avec la sociologie : Travail et régulations sociales ; Impact de l'analyse du travail sur les relations sociales; Tensions entre activités professionnelles et décision organisationnelle ; L'autonomie dans le travail.

- En rapport avec la physiologie : Évaluation de l'astreinte thermique à l'aide de la fréquence cardiaque ; Affections vertébrales dans les travaux du bâtiment; Effets du travail de nuit en milieu hospitalier; Confort thermique dans le travail tropical.

- Le même type d'illustration pourrait être trouvé avec d'autres disciplines.

On pourrait caractériser ces recherches en disant qu'elles sont susceptibles d'un double label : ergonomique et de la discipline associée. Par exemple, une recherche sur l'exploration visuelle menée en situation de travail est indexable à la fois en ergonomie (si elle est associée ou intégrée à une étude à finalité ergonomique) et en psychologie (en tant qu'elle enrichit le chapitre de l'exploration visuelle en montrant les modifications éventuelles des types de mécanismes de celle-ci dans une situation d'un type particulier).

On peut préciser ce qui fait la différence entre une recherche typiquement ergonomique et une recherche à double label en prenant l'exemple du double label ergonomie-psychologie, dans le domaine de l'exploration visuelle, bien illustré par les articles d'un numéro spécial du Travail 
Humain $(1983,46,1)$ consacré à «l'exploration visuelle dans le travail ». La recherche à double label a en général un label dominant : s'il est psychologique, cela signifie que la recherche est orientée prioritairement par une problématique psychologique. Elle vise d'abord à s'inscrire dans le cadre de recherches conduites en psychologie sur le thème choisi. Elle se référera prioritairement à des recherches théoriques auxquelles elle empruntera ses hypothèses. Par exemple, des recherches se sont intéressées à la contribution du champ périphérique à la saisie visuelle dans la situation de conduite automobile. Elles s'appuient sur les connaissances théoriques et méthodologiques déjà développées pour l'étude de la vision périphérique auxquelles elles apporteront, d'ailleurs, des compléments utiles (Hella, 1983). Le label dominant pourra aussi être ergonomique. Ainsi le point de départ d'une telle recherche pourra viser l'amélioration des conditions d'un travail particulier dans lequel il sera apparu que les connaissances et techniques de l'exploration visuelle peuvent apporter des éléments d'information importants. Ce sera le cas, par exemple, pour l'étude du rôle des horaires de travail dans une activité de surveillance ou dans le travail de tractoriste, ou encore pour l'étude de clichés radiographiques (exemples discutés dans le même numéro).

La recherche à double label à dominante ergonomique fait prévaloir la coordination de différents points de vue issus des disciplines associées ${ }^{1}$. Cette différence d'accent se traduit par le fait que, dans un cas, on conclura en marquant l'intérêt de l'hypothèse disciplinaire pour l'étude du problème pratique alors que dans le second, on s'attachera surtout à souligner l'avantage d'articuler ce point de vue prioritaire avec d'autres pour une meilleure solution du problème. Dans le premier cas, la mise en œuvre d'une solution concrète sera souvent évoquée, mais rarement poursuivie. En revanche, dans le second cas, cette mise en œuvre revêtira un rôle majeur.

Les recherches à double label représentent pour l'ergonomie une manière d'élargir son champ en mettant à profit une meilleure exploitation des disciplines associables. Cette démarche constitue une des voies d'élaboration du domaine des connaissances spécifiquement ergonomiques. On pourrait parler d'une migration des connaissances disciplinaires associées vers des connaissances ergonomiques. Au départ, on dira que telle recherche ergonomique emprunte des connaissances au domaine de la discipline associée et, ensuite, que progressivement, ces dernières connaissances sont intégrées à l'ergonomie. Pour reprendre un exemple précédent, on a d'abord cherché en psychologie les connaissances sur l'exploration visuelle pertinente à la conduite automobile, mais très vite, celles-ci se sont intégrées au problème ergonomique qu'elles ont éclairé, par exemple, la prise d'information en situation de virage ou d'intersection.

\section{5.- Connaissances relatives aux interventions}

Ces études sont celles qu'on trouve majoritairement dans les congrès, colloques et séminaires, et qui figurent dans les Actes de ces réunions. Il en sera largement question dans la troisième partie. Elles constituent des matériaux de base de la discipline et peuvent être exploitées à des fins diverses. Pour celui qui les rédige, elles sont, parfois, la première phase d'un travail d'élaboration plus systématique et plus approfondi pour être ainsi, par exemple, intégrée à un mémoire ou à une thèse. Ces études sont souvent aussi très intéressantes en tant qu'elles donnent une idée de l'ergonomie vivante, de son développement. On y voit apparaître des problèmes nouveaux en même temps que des manières nouvelles d'aborder et de traiter les questions. Les études issues d'intervention aident à dresser un état de la discipline à un moment donné. Elles peuvent être la source d'échanges et de rapprochement entre ergonomes travaillant sur des thèmes voisins. Ce genre de textes est en général très révélateur de la compétence de leur auteur et ce serait une erreur de penser qu'ils sont toujours faciles à rédiger. Ils doivent notamment éviter deux écueils : (1) celui de raconter l'intervention en s'égarant

1. Ceci ne doit pas donner à penser que l'ergonomie ne se définit que par cette démarche interdisciplinaire : elle utilise cette démarche à ses fins propres. 
dans les détails de la technologie support de l'activité étudiée ; (2) celui de noyer le travail concret dans un tissu de généralités lui faisant perdre toute originalité, voire tout intérêt. Le cadre définissant la problématique, et le traitement des données recueillies, doivent être articulés et équilibrés, car les insuffisances de l'un ne peuvent être compensées par le volume exagéré de l'autre.

\section{6.- Les connaissances en méthodologie}

Les connaissances en méthodologie ont été importées le plus souvent d'autres disciplines, mais adaptées aux caractéristiques des situations auxquelles s'intéresse l'ergonomie. Une des caractéristiques essentielles de celles-ci est liée au fait qu'elles s'imposent à l'ergonome et que les possibilités de les modifier à des fins d'analyse sont très réduites. On ne peut que rappeler ici les débats sur les rapports entre études de laboratoire et études sur le terrain. L'adaptation peut être assez directe et on reconnaîtra souvent dans les méthodes utilisées en ergonomie les méthodes pratiquées dans d'autres disciplines. Mais les études ergonomiques, face aux contraintes auxquelles elles sont soumises et en fonction des perspectives théoriques dans lesquelles elles s'inscrivent, ont aussi amené à enrichir les méthodes. Citons, par exemple, les méthodes d'auto-confrontation simple et croisée, les méthodes d'analyse de séquences d'états et d'événements, les méthodes de simulation en vue de la recherche et de la formation, les méthodes d'analyse des activités collectives, etc.

L'originalité des méthodes réside aussi dans la conjugaison de différentes techniques, appelée par la nécessité de saisir les interactions des différentes composantes de l'activité de travail ; elle est liée aussi au statut des travailleurs qui ne sont pas des sujets passifs d'expérience, mais parties prenantes de l'intervention.

Un exemple de publication de synthèse dans ce domaine est celui de Bisseret, Sébillotte et Falzon (1999) qui rassemble un certain nombre de «techniques pratiques pour l'étude des activités expertes » sous une forme très opérationnelle.

\section{7.- Connaissances relatives aux pratiques d'interventions}

Dans cette catégorie figureraient des connaissances à caractère transversal, se rapportant à des questions susceptibles de se poser dans des interventions diverses. La meilleure illustration qu'on puisse en donner serait à emprunter aux thèmes des Journées de Bordeaux organisées par Daniellou (2003a). Voici quelques exemples :

- L'ergonomie et les compromis

- La construction de l'intervention ergonomique

- L'ergonomie et les compétences

- Les prescripteurs et les prescriptions

\section{8.- Connaissances centrées sur l'analyse de l'activité de l'ergonome}

Pendant longtemps, la pratique a été conçue comme l'application des connaissances scientifiques du domaine concerné. Cette vue erronée a été mise en question, notamment par Schön $(1983,1987)$ qui a montré que la mise en œuvre des connaissances constituait une compétence qui débordait les connaissances elles-mêmes. Il parle d'un « hiatus entre les connaissances professionnelles et les exigences de la pratique dans le monde réel » $(1983$, p. 45). Il fait remarquer que longtemps on a conçu à tort la pratique comme un processus de résolution de problème ("problem solving ») en ignorant qu'elle était aussi un processus de position de problème (" problem setting ») (p. 40) par lequel une situation problématique est transformée en un problème, c'est-à-dire par lequel on « nomme les choses à considérer et le cadre dans lequel on veut les traiter » (id.). Schön est l'un de ceux qui ont suscité un courant d'intérêt pour l'analyse des activités des praticiens. La pratique en ergonomie n'a pas 
échappé à ce courant. Un exemple typique en est fourni par l'ouvrage que Lamonde (2000) a consacré à l'analyse de "la pratique ergonomique» conçue comme "un regard sur la pratique professionnelle ». Les publications sur ce thème devraient se développer pour faire valoir la spécificité de la compétence du praticien, l'expliciter et contribuer ainsi à faire disparaitre cette opinion que Schön dénonce à juste titre et selon laquelle il y aurait une sorte de hiérarchie sociale entre les représentants des « trois composantes des connaissances professionnelles : les connaissances scientifiques de base, les connaissances appliquées et les pratiques qui mettent en jeu ces connaissances pour leur propre fin » (1983, p. 24). Il s'agit plutôt de distinguer des fonctions - acquisition et organisation des connaissances, intervention pratique, etc. En ce qui concerne la pratique, le problème sera d'élucider la nature du savoir, souvent tacite, mis en jeu pour pouvoir le communiquer et l'enrichir.

\section{2.- L'expérience acquise dans les interventions}

Ces dernières années ont vu apparaître beaucoup de réflexions sur la nature de l'expérience, sur ce qu'on acquiert dans l'exercice d'une activité et par cet exercice, qui n'était pas dans le lot des connaissances acquises initialement. Schön (1983) parle d'un fossé («gap ») entre les connaissances professionnelles et les exigences de la pratique dans le monde réel (p. 45). Cette expérience est considérée souvent comme indicible, comme une compétence tacite dont celui qui la possède ne peut toujours apporter de justifications cohérentes, d'où l'allusion souvent faite à l'art du praticien. Wisner (1995) écrivait qu' " il faut peut-être se résoudre à accepter que l'ergonomie ne soit pas une science, mais un art nourri de méthodes et de connaissances issues de la recherche scientifique... » (p. 125). On trouve cette même allusion à l'art chez Schön (1983) pour décrire des aspects des activités du praticien. Il est encore important de réfléchir sur la nature de l'expérience, quoique les idées essentielles ont été exprimées et discutées dans plusieurs textes : nous nous référerons ici surtout à ceux de Schön $(1983,1987)$ et de Daniellou (2003b). Pour aborder ces questions, on examinera d'abord les rapports entre connaissances professionnelles et compétences professionnelles, ensuite les rapports entre expérience et compétences tacites ou incorporées. Enfin, on discutera de la place des compétences sociales.

\section{1.- Les compétences professionnelles ne sont pas faites que de connaissances professionnelles}

On entend par connaissances professionnelles celles qui sont très directement liées à la profession, cette part invariante des connaissances qui sont à mettre en œuvre quelles que soient les conditions d'exercice de la profession : ce sont, en particulier, celles qui sont enseignées au cours de la formation. Considérons, par exemple, le cas d'un enseignant : ses compétences sont liées aux connaissances qu'il possède de sa discipline, à sa manière de les organiser, à ses schémas d'enseignement. Mais son activité quotidienne d'enseignant dépend aussi des finalités qu'il donne à son travail, du milieu dans lequel il l'exerce, de ses collègues, des installations scolaires, etc. La manière d'affronter ces conditions variables n'est pas précisément enseignée. Il en est de même pour l'ergonome qui conçoit une intervention. Il ne s'agit pas pour lui d'appliquer un schéma tout fait à une situation bien catégorisée, mais de prendre la mesure de la situation dans sa complexité, humaine, technique, organisationnelle, d'évaluer comment obtenir l'adhésion des responsables et la coopération des utilisateurs. L'enseignant peut très bien connaître son domaine et organiser son enseignement sans obtenir l'adhésion et la coopération nécessaires des élèves. De même l'ergonome peut avoir fait une très bonne analyse du problème sans réussir à mettre en œuvre une solution qui obtienne un fort courant d'adhésion. On trouve d'excellentes réflexions sur ce point dans le livre de Martin et Baradat (2003). Dans la partie « la construction de l'intervention en ergonomie », Daniellou (2003c) y note justement qu' « en tout point de l'entreprise se croisent une diversité de logiques ». Il montre que 
toute intervention s'insère dans un milieu complexe et qu'elle est susceptible de provoquer des effets que l'ergonome doit s'attacher à gérer. Citant Carbaledda, il écrit que « l'ergonome a donc besoin, quand il s'apprête à concevoir ou à transformer une situation de travail, d'identifier les différentes catégories professionnelles du champ d'activité duquel elle relève, mais aussi les différentes logiques de l'entreprise qui se croisent dans cette situation » (p. 139). Aux franges de l'exercice de toute profession se trouvent donc des activités qui ne sont pas forcément spécifiques de cette profession, mais qui sont souvent essentielles à la réussite des activités proprement professionnelles. Ces activités « latérales » ne font généralement pas l'objet d'une formation formelle, mais leur maitrise est laissée à la pratique. Cette situation n'est pas totalement inéluctable et on peut penser qu'une formation efficace devrait prendre en compte ces conditions d'exercice de la profession et leur accorder un statut à la mesure de leur importance. Dans cette frange, tout n'est peut-être pas enseignable, mais on peut faciliter l'acquisition de ce qui ne l'est pas. Il ne faut pas admettre trop vite, comme on l'entend souvent, que «cela ne peut s'acquérir que par la pratique ». C'est par l'analyse et par une meilleure connaissance de l'activité du praticien que peut être explicitée cette partie de la compétence qui déborde les connaissances classiquement enseignées. C'est à quoi visent d'ailleurs les formules de stage et les pratiques d'initiation suivies par un professionnel. Mais on peut sans doute, parfois, aller plus loin en explicitant des procédures d'analyse et d'intervention qui induisent une certaine manière de poser et de traiter les problèmes. Cette démarche est étroitement liée aux efforts faits pour catégoriser des types de problèmes en fonction de l'intervention. Elle sera favorisée par l'effort du praticien lui-même pour prendre conscience de sa propre démarche et l'analyser.

\section{2.- L'expérience recouvre une part importante de compétences tacites}

L'un de nous a eu l'occasion de discuter la notion de compétence incorporée, synonyme de celle de compétence tacite (Leplat, 1997a) et nous nous contenterons d'examiner ici comment la présente Schön (1983) : « Souvent, nous ne pouvons pas dire ce que nous savons » (p. 49) et corrélativement, «nous connaissons plus que nous pouvons le dire» (p. 51). Il cite cette phrase de Barnard selon laquelle des hommes d'expérience « ne pourraient pas écrire les manuels qu'ils ont enregistrés dans leur tête » (p. 52). Il nomme cette expérience « connaissance-en-action » (knowing-in-action) et la considère comme le « mode de connaissance pratique ordinaire » (p. 54).

La discussion précédente renvoie au problème de l'identification et de l'explicitation des compétences. À cet usage, on pourrait proposer une classification des compétences en trois catégories :

1. Les compétences reposant sur des connaissances extériorisées directement exploitables, ou sur des documents orientés vers la pratique (par exemple, notice d'utilisation, procédure écrite d'exécution de contrôle).

2. Les compétences reposant sur l'intériorisation des connaissances précédentes. Il n'est plus besoin de consulter les documents précédents qui ont été mémorisés et rendus ainsi immédiatement disponibles.

3. Les compétences qui reposent sur des acquisitions pratiques non préalablement explicitées. Ce sont des connaissances liées à l'action, qui adhèrent à cette action et qui s'expriment par elle. Ce sont les « connaissances-en-action » évoquées plus haut.

Ce sont ces deux derniers types de compétences qui ont été souvent désignés sous le nom de compétences incorporées. Elles sont caractérisables par les propriétés suivantes : elles sont facilement accessibles, peu coûteuses sur le plan de la charge mentale, très liées au contexte, difficilement dissociables.

Cette classification fait apparaître deux catégories de compétences incorporées : l'une résultant de l'intériorisation de compétences initialement explicitées, l'autre de compétences acquises par l'exé- 
cution des actions. Une propriété de ces compétences incorporées qui ne recoupe pas cette distinction fondée sur l'origine est celle du caractère explicitable. Les compétences de la dernière catégorie sont en général difficilement verbalisables, celles de l'autre catégorie, fondées initialement sur l'intériorisation de connaissances verbalisables, le sont plus facilement mais pas toujours. En effet, avec un usage répété, elle peuvent devenir parfaitement intériorisées et ne plus se révéler que dans l'action : le sujet agit correctement, mais il ne sait plus exprimer ce qu'il fait autrement que par cette action, et plus par le langage. Dans d'autres cas, quoique ayant bien intériorisé son action, il continue à pouvoir en justifier. En cas d'incident, par exemple, il peut trouver les éléments d'analyse de cet incident qui lui permettront d'élaborer une solution originale. Les routines intellectuelles, comme les autres, ont un rôle ambigu : d'une part, elles permettent un fonctionnement économique grâce auquel des compétences plus larges peuvent être acquises : quand on a bien assimilé les questions à poser pour l'analyse d'un type de travail, on peut alors être plus attentif à des faits qui risquent d'échapper à celui qui est focalisé sur son schéma d'entretien et a peur d'oublier des éléments d'information. Mais, d'autre part, ces routines sont un facteur de sclérose susceptible de figer l'activité et de lui faire perdre progressivement son caractère adaptatif. Comme le dit Schön (1983), lorsque les routines prennent une importance trop grande, «le praticien peut manquer d'importantes occasions de réfléchir sur ce qu'il est en train de faire (...) il est entraîné dans des patterns d'erreur qu'il ne peut pas corriger (...). Et si, comme cela arrive souvent, il apprend à être sélectivement inattentif aux phénomènes qui n'entrent pas dans ses catégories de connaissances-dans-l'action » ( $\mathrm{p}$. 61), cela peut entraîner étroitesse d'esprit et rigidité. L'expérience acquise dans un champ étroit risque toujours de se scléroser et l'agent peut même perdre la justification de ses actions, qu'il possédait initialement : il fait des choses sans bien pouvoir en rendre compte.

Pour que les compétences incorporées restent les composantes essentielles de l'expérience et pour éviter l'installation précoce de ces routines, comme pour pallier leurs inconvénients, l'ergonomie a proposé plusieurs types de remèdes qu'elle peut s'appliquer à elle-même. On en signalera quelques uns à titre de rappel : élargir le champ et la variété des problèmes à traiter, être ouvert aux discussions avec les interlocuteurs, participer à des débats sur des questions professionnelles, rédiger des communications pour se soumettre à l'évaluation et à la critique de ses pairs, etc. Il y a toujours un équilibre difficile à trouver entre des compétences solidement installées et immédiatement opérationnelles, et des compétences ouvertes, plus difficiles à mettre en œuvre, mais susceptibles de répondre à des problèmes nouveaux de manière originale et efficace.

\section{3.- Les compétences sociales requises par l'intervention}

L'intervention exige la coopération de ceux dont elle est appelée à transformer l'activité. L'ergonome est conduit à s'impliquer dans les situations s'il veut contribuer efficacement à leur transformation. En s'impliquant dans la situation, c'est-à-dire en coopérant avec les intéressés à l'élaboration et à la modification de la situation, il suscite des regards nouveaux sur celle-ci, en même temps qu'il enrichit les siens. C'est par ces dialogues que peut se créer un espace dans lequel deviendront plus lisibles les actions de chacun et que pourra se développer une dynamique en faveur des modifications. L'intervention devient alors une coaction des différentes personnes impliquées avec l'ergonome. De son côté, ce dernier est amené à concevoir son action non plus seulement à partir de la seule analyse initiale du travail, mais à partir de la confrontation de ses propres modèles avec ceux de membres de l'entreprise. L'intervention devient alors une construction collective où le rôle de l'ergonome est important en tant qu'il peut aider à la communication des personnes impliquées en leur proposant notamment un langage et des modèles utiles. Dans cette perspective, l'analyse du travail, qui conduit au diagnostic, et l'intervention s'élaborent progressivement en interagissant et en s'enrichissant réciproquement au cours de l'action. À cette fin, le rôle de l'ergonome requiert des compétences sociales, entendant par là des compétences pour l'organisation des dialogues et des coopérations. En ce sens, on peut dire que les compétences sociales, acquises en 
grande partie au cours des interventions, constituent une composante importante quoique souvent tacite des compétences générales de l'ergonome praticien.

\section{3.- Les connaissances produites dans les interventions et leur capitalisation}

L'ensemble des connaissances susceptibles de concourir à la conception et à l'aménagement de situations de travail combine les apports de trois sortes d'investigations différentes : interventions à visée pratique, études et recherches menées en milieu de travail avec une visée de production de connaissance, sans visée pratique immédiate, études hors-milieu de travail.

On tentera de situer ici l'apport propre des analyses réalisées dans un cadre d'intervention, leurs conditions de production, leurs atouts et leurs contraintes. Mais il importe de noter en préalable que la distinction intervention / étude en milieu de travail est à compléter par une distinction en fonction des cadres professionnels dans lesquels s'inscrivent les auteurs de ces opérations : organismes de consultants externes / services internes d'ergonomie / organismes de recherche. En effet ces deux distinctions se recoupent dans une large mesure mais pas totalement : un intervenant peut de temps à autre réaliser une étude sans visée d'aménagement et un chercheur une intervention. Mais les cadres professionnels dans lesquels s'inscrivent les uns et les autres ne sont pas sans incidence sur leurs types de production, quelle que soit la nature de l'action dans laquelle ils sont engagés.

\section{1.- Atouts et limites des interventions dans la production des connaissances ergonomiques}

Les interventions ont un atout essentiel qui est d'être en prise directe avec la réalité des situations de travail dans toute leur complexité et leur spécificité. Il faut noter cependant qu'elles n'en ont pas le monopole : les études menées sur le terrain avec une visée de connaissance mais sans finalité d'aménagement ont aussi cet avantage. Mais les conditions dans lesquelles les unes et les autres peuvent tirer parti de cet atout sont différentes à plusieurs égards.

Parmi les situations de travail qui font l'objet d'une intervention, il est fréquent que certaines offrent des possibilités d'examiner une condition d'activité jusqu'alors méconnue ou peu documentée, mais qui n'est pas l'objet de la demande. Face à une telle situation la possibilité, pour un intervenant, de contribuer au progrès de la connaissance de cette condition d'activité dépend pour partie du poids de cet élément dans le problème qui a motivé la demande d'intervention. S'il est majeur (ou apparaît comme tel au demandeur) l'espace est ouvert; si par contre il apparaît comme un élément secondaire, l'intervenant et le demandeur seront sans doute plutôt poussés à s'en tenir à une analyse minimale, étant donné par ailleurs les contraintes de temps et de ressources inhérentes à toute intervention. Daniellou (1998) décrit très bien comment « toute la diversité des entreprises, des conditions des interventions, des histoires des acteurs de l'entreprise et des ergonomes va donner lieu à de multiples variations » concernant l'arbitrage jugé le plus stratégique entre la conduite du travail de diagnostic et celui de l'amorce d'actions de changement.

Face à cette même situation, une étude sans finalité pratique peut avoir les coudées plus franches, et pousser plus loin l'analyse de cette condition qui pourra contribuer à en éclairer le jeu dans d'autres situations où son poids relatif serait différent. Ainsi, par exemple, a-t-on pu se saisir d'une demande du Service des Douanes de procéder à une évaluation comparative des divers systèmes d'horaires pratiqués dans l'ensemble des brigades couvrant le territoire national pour y inclure un axe portant sur les effets combinés des horaires postés et du contenu du travail (Prunier-Poulmaire \& Gadbois, 1998). 
La production de connaissances nouvelles, dans le cadre d'interventions, est par ailleurs très dépendante de la connaissance que les intervenants ont de l'état présent du savoir concernant les phénomènes en jeu dans la situation de travail qu'ils ont pour mission d'aménager. Le savoir acquis au cours de la formation initiale, bien qu'enrichi au fil de l'expérience, des lectures, des congrès et des rencontres professionnelles, peut néanmoins ne pas être pleinement en phase avec les dernières avancées dans un domaine particulier. Ainsi, discutant des TMS, Vézina (2001) s'interroge-t-elle : « l'attention n'est-elle pas mobilisée et limitée par les facteurs biomécaniques que l'on retrouve de façon classique dans les grilles d'observation communément utilisées ? les ergonomes ont-ils toujours la formation suffisante pour bien analyser le travail physique et comprendre la sollicitation musculo-squelettique nécessaire à l'exécution des opérations observées ? ».

La possibilité de percevoir les opportunités offertes par une situation de travail de pousser l'exploration de questions irrésolues, en particulier lorsqu'elles ne sont pas centrales par rapport aux préoccupations des demandeurs, suppose d'avoir des connaissances assez larges et actualisées permettant de repérer ces questions. Or, souvent, les conditions d'exercice du métier d'intervenant (temps disponible, délais, calendriers, accès matériel aux sources documentaires) ne sont pas des plus propices au maintien d'une telle veille. Certes comme le relève Mazeau (2003) « il existe une littérature scientifique abondante pour nourrir (la) réflexion à peu près sur tous les types de tâches, dans tous les domaines,... il est bien rare que (l'on) ne trouve pas de littérature sur le sujet aidant à structurer mieux ou plus rapidement une problématique ». Mais il note en même temps que ces "montagnes d'information », accessibles en particulier par internet, "n'ont aucune utilité lorsque ne préexiste pas une expertise du domaine ». Et les résultats de l'enquête faite par l'un de nous sur les références citées par les auteurs des communications présentées aux Congrès de la SELF (Leplat, 1997b) tendraient à montrer que les conditions d'irrigation des interventions par les recherches les plus récentes ne sont sans doute pas si aisées. Il nous semble en particulier que manque actuellement un chaînon intermédiaire que constitueraient des revues de questions périodiques, fréquentes, fournissant des synthèses sur les avancées scientifiques récentes et mettant l'accent sur les apports potentiels aux pratiques d'intervention; il devrait y avoir là une contribution des chercheurs et des enseignants-chercheurs qui soit institutionnellement encouragée, et un rôle qui est actuellement trop peu rempli par les revues francophones. C'est une des fonctions qu'on peut espérer voir prise en charge par une revue telle qu'«@ctivités».

Il faut noter aussi que l'accès des praticiens aux sources d'informations sur l'évolution des connaissances passe par ailleurs par les contacts personnels dont ils peuvent disposer dans les milieux qui détiennent cette information (universités, centres de recherches, organismes de diffusion). Sur ce point il est manifeste qu'existent de grandes différences interindividuelles qui sont sans doute à rapporter en partie aux circonstances de la formation et aux conditions d'exercice du métier (notamment géographiques). Mais de façon générale, il semble que le capital relationnel soit parfois limité et la question se pose de savoir par quelles voies la situation pourrait être améliorée.

Dans le cadre des interventions l'identification et la mise en lumière du jeu des déterminants de l'activité peuvent être plus ou moins poussées, selon les ressources (temps, finances, compétences, ...) allouées à l'intervention et selon les caractéristiques du terrain constituant le champ d'investigation (extension et diversité de la gamme des postes de travail et de la population des opérateurs au regard des variables pertinentes). Selon ce qu'il en est, une intervention peut produire des éléments de connaissances ayant un plus ou moins grand degré de généralité. Plus nombreuses seront les sources de variations qu'il aura été possible d'examiner, mieux délimité sera le champ de validité des résultats obtenus. Ainsi, parmi les enseignements issus des analyses réalisées sur les postes d'une chaîne d'abattage de porcs (Abisou, 1993), il se peut que certains valent uniquement pour d'autres postes de travail utilisant les mêmes outils sur un système et dans des conditions identiques ; mais y en a-t-il qui vaillent plus largement, pour toute chaîne d'abattage de porcs ? pour toute chaîne d'abattage? pour tout travail à la chaîne ? Dans une entreprise fonctionnant en continu 
dont la plupart des salariés ont entre 30 et 40 ans, si l'adoption d'un système d'horaires à rotation rapide a été jugé positivement, dans quelle mesure peut-on pronostiquer que le recours à une alternance rapide aura des résultats analogues dans d'autres entreprises où la majorité des salariés ont plus de 40 ans ? De la même manière les résultats acquis sur les contraintes présentes aux postes de caissières d'un hypermarché (Prunier-Poulmaire, 1998) sont ils directement applicables aux caisses de moins de 10 articles comme à celles équipées d'un dispositif d'ensachage manié par la caissière ? Dans quelle mesure et comment les connaissances considérées comme générales, (par exemple concernant les angles articulaires les plus pénalisants), sont elles modulées par ces particularités ou par d'autres facteurs tels que la durée du travail, l'organisation des pauses, les activités amont ou aval ?

Que l'on se trouve dans le cadre d'une intervention ou dans celui d'une étude sans finalité immédiate d'action, la capacité à répondre à ces questions est largement circonscrite par les caractéristiques du terrain. La situation est sans doute plus difficile dans le cadre des interventions pour lesquelles l'élaboration et la mise en place d'aménagement est l'objectif essentiel, la réponse à des questions générales, bénéfice secondaire, n'étant acquise que par surcroît. Comme le rappelle Falzon (1997), l'analyse de cas, qui est le lot général des interventions, peut être inspirée par deux visées opposées : une visée généralisante où l'objectif est d'emblée d'aboutir à des résultats exportables à d'autres situations (ce qui suppose d'intégrer dans la conduite de l'intervention une certaine part d'activité métafonctionnelle); une visée particularisante où on cherche à décrire dans le détail la spécificité de la situation étudiée. Comme l'a montré l'un de nous à l'occasion d'une réflexion sur la méthode clinique en ergonomie (Leplat, 1997c), c'est bien dans cette seconde perspective que se trouvent les praticiens : ayant à trouver la solution des problèmes posés par le cas, il leur faut se focaliser sur celui-ci. Cette opposition n'est cependant pas radicale. Il faut en effet remarquer avec Falzon que « les faits scientifiques ne sont pas établis sur la base d'une expérimentation unique mais plutôt en répliquant une expérimentation dans différentes conditions ». Les interventions peuvent constituer, dans une certaine mesure, une pratique collective de réplications, même si elles ne sont pas réalisées selon une programmation raisonnée mais se développent plutôt au fil des circonstances.

\section{2.- Les contributions des interventions à l'avancée des connaissances ergonomiques}

L'analyse des situations de travail menée par les ergonomes dans le cadre d'interventions apporte des connaissances nouvelles, absentes souvent des corpus disciplinaires des sciences « mères », et qui peuvent être :

- des connaissances relevant d'une de celles-ci et portant sur des facteurs (ou des modalités de facteurs) non considérés jusqu'alors dans son champ, en raison de leur absence dans l'univers de référence des chercheurs de la discipline, ou bien parce que la dynamique du développement théorique, sur la base des paradigmes expérimentaux utilisés orientait vers d'autres voies ;

- ou bien des connaissances concernant les effets de combinaison de facteurs relevant de disciplines différentes et de ce fait pris en compte par aucune d'elles. Ces connaissances nouvelles contribuent à élargir le champ d'analyse couvert par les disciplines mères et par l'ergonomie, selon trois axes principaux.

- Tout d'abord quant à l'identification des exigences intrinsèques des tâches : par exemple, on sait combien celles-ci sont imparfaitement explorées par les disciplines mères qui modélisent l'activité essentiellement à partir des caractéristiques de l'organisme (pour la bio-mécanique par exemple : muscles, articulations, os, tendons) plutôt que des caractéristiques de la mise en jeu de ces éléments dans les situations de travail. Ce que souligne par exemple Vézina (2001) en relevant que la contraction musculaire des régions permettant de mieux stabiliser le mouvement dans les tâches requérant vitesse et précision, est un facteur critique dans les postes de 
montage sur chaîne automobile, mais qu'il n'est pas toujours présent dans les grilles d'observation souvent utilisées dans le cas de tâches manuelles, et que les exigences de mouvements qui doivent se faire en force tout en étant retenus, rencontrées sur certains postes de fabrication automobile, ont jusqu'ici rarement été évoquées.

De façon très générale, la diversité et la complexité des situations de travail font que la plupart des interventions contribuent à l'élargissement et à l'approfondissement de la catégorisation. Ces avancées ne vont pourtant pas de soi, étant donné les «ifficultés courantes à penser sa propre situation de travail ou celles que l'on côtoie au quotidien » que rappelle Daniellou (1998), et qui résultent de 3 familles de mécanismes : « difficultés de nature cognitive à mettre en mots des compétences inscrites dans le corps mais qui n'ont jamais fait l'objet d'une formalisation et d'un énoncé, (...) poids des descriptions sociales dominantes, (...) défenses mises en place par les travailleurs pour supporter des situations qui sont source de souffrance ». Ces difficultés, déjà évoquées dans la partie 2, ne peuvent être dépassées sans un travail systématique auquel, le plus souvent, seuls peuvent inciter les enjeux liés aux interventions.

- Un second axe d'élargissement de l'éventail des déterminants de l'activité a trait aux exigences de la tâche liées à l'inscription du poste de travail dans un processus de production et aux modes d'organisation de celui-ci. Ainsi, par exemple, un boucher sur une chaîne d'abattage ne doit pas seulement satisfaire à l'objectif de la tâche assignée à son poste mais aussi transmettre la carcasse sur laquelle il intervient dans un état qui ne complique pas la tâche de l'opérateur qui occupe le poste suivant : effectuer l'opération de découpe du cuir ou du muscle qui lui revient en propre mais de telle sorte que l'opérateur suivant puisse trouver les meilleures prises sur la carcasse et la meilleure entame (Bourgeois et al., 2000) ... Or cette exigence ne figure pas dans le cahier des charges de conception du poste de découpe, et ses implications ne sont pas nécessairement évidentes pour tout autre que l'opérateur qui aura à gérer cette exigence. De tels déterminants ne peuvent intervenir que dans les milieux de travail et ne peuvent donc être considérés par des modèles conçus sans référence étroite à ces milieux-là. Mais, par contre, la visée pragmatique des interventions fait que celles-ci ne sauraient les laisser de côté.

- L'élargissement des analyses dû aux interventions, également lié à leur visée pragmatique, porte en troisième lieu sur des déterminants d'ordre supérieur qui ne font pas partie des caractéristiques du poste de travail mais influent sur celles-ci, et tiennent aux conditions générales de la gestion des conditions de travail au sein de l'entreprise. Citons à titre d'exemple les sous-effectifs de personnel infirmier auxquels sont confrontés actuellement les hôpitaux français, et qui résultent des choix gouvernementaux en matière de création d'emplois hospitaliers et de réduction du temps de travail à 35 heures. Ou encore l'analyse proposée par Daniellou (1998) de l'apparition des TMS comme résultante du blocage, chez différents acteurs de l'entreprise, de la dynamique qui devrait exister entre les 3 pôles suivants : pouvoir agir, pouvoir penser, pouvoir débattre. Ce blocage se manifeste par « des boucles infernales qui comportent 3 composantes :

- une source non détectée par l'entreprise de perte de productivité ;

- une recherche de compensation de cette perte de productivité par une pression directe sur les cadences ou les effectifs ;

- une aggravation de la perte de productivité par les effets secondaires de cette pression ».

L'exploration de ce niveau d'analyse, touchant aux relations entre les contraintes organisationnelles générales et l'organisation des conditions de travail est clairement un territoire propre de l'ergonomie, tout à fait distinct de ceux des deux disciplines-mères majeures physiologie et psychologie - dont elle est issue, et à propos duquel elle devrait avoir à dialoguer avec la sociologie et la psycho-sociologie du travail et des organisations. 


\section{3.- Des expériences personnelles au savoir collectif : la capitalisation}

Les interventions ont le privilège d'être confrontées à la complexité des situations de travail et d'être par là même en mesure d'en mettre en lumière des aspects souvent cruciaux et néanmoins méconnus. L'identification d'un facteur critique non repéré jusqu'alors est ainsi l'apport premier de bon nombre d'interventions. Au-delà de ce premier pas, selon les conditions qui président à leur déroulement (notamment quant au temps et aux ressources imparties), les interventions sont plus ou moins en mesure de pousser leurs analyses pour établir des modèles ayant un certain degré de précision ; ainsi, allant au-delà d'hypothèses du type entrées / sorties, elles peuvent plus ou moins contribuer à spécifier le mécanisme à travers lequel le facteur critique identifié détermine l'activité, spécifier ses interactions avec d'autres facteurs, évaluer l'ampleur de ses effets, estimer des seuils de confort et d'efficacité.

Comme on peut s'en rendre compte à la lecture des Actes des Congrès de la SELF, toutes les interventions ne sont pas en situation de remplir complètement ce programme; elles ne sont d'ailleurs pas non plus toujours dans la nécessité de le faire. Mais, comme pour tous les domaines de la connaissance, la constitution du savoir ergonomique est un processus collectif reposant sur le cumul d'investigations particulières. Et si l'on ne saurait espérer que les contraintes qui pèsent sur l'élaboration de connaissances nouvelles dans le cadre de la majorité des interventions puisse changer notablement dans l'immédiat, il nous paraît, par contre, possible d'agir sur les conditions de mise en circulation des apports des interventions et de leur intégration dans le corpus général des connaissances ergonomiques.

L'intégration des connaissances nouvelles acquises dans le cadre des interventions passe naturellement par un travail d'écriture, de publication et de diffusion qui a inévitablement des contraintes et des coûts mais dont la réalisation pourrait être davantage soutenue par la communauté à travers divers moyens, à différents niveaux.

La première étape est celle de la formalisation des enseignements issus de l'intervention, qui s'opère le plus souvent d'abord à travers la rédaction d'un texte de conclusions à destination des parties prenantes de l'intervention (commanditaires et salariés concernés). Ce texte a une vocation opérationnelle dans l'intervention dont l'objectif est d'aboutir à un aménagement des conditions de travail, ce qui en commande le fond et la forme. Les résultats des analyses réalisées y sont retenus et présentés en fonction de leur utilité par rapport à l'objectif. Par ailleurs, on peut se dispenser d'y faire état de certaines informations sur la situation-problème et son contexte dans la mesure où tous les lecteurs, appartenant à l'entreprise, les connaissent (ce qui, à l'expérience, demande toujours à être vérifié, et souvent à compléter ou corriger).

Le contenu de ce texte, plus éventuellement d'autres constats ou enseignements tirés par les intervenants mais non inclus dans le rapport, viennent enrichir le « savoir d'expérience » de ceux-ci. Mais il s'agit encore là d'un savoir privé et la diffusion du rapport ne dépasse guère, généralement, les frontières de l'entreprise. Diffuser vers la communauté des ergonomes les connaissances nouvelles issues d'une intervention suppose donc de rédiger un second texte, différent dans la forme et dans le fond. Dans le fond notamment parce qu'il peut être plus précis sur des aspects de méthode, sur certains résultats, et silencieux sur d'autres n'apportant rien de nouveau, et parce qu'il doit situer les apports originaux par rapport au corpus des connaissances existantes.

La rédaction de ce second texte demande donc évidemment un travail supplémentaire, en particulier parce que l'articulation des acquis de l'intervention avec les connaissances existantes requiert un travail spécifique. Or c'est là un investissement apparemment " gratuit » sur le moment, consommateur de temps, qui n'est pas aisément compatible avec les contraintes économiques qu'ont à gérer les consultants. De ce fait une partie des connaissances produites dans le cadre d'interventions reste du domaine privé des « savoirs d'expérience » personnels alors qu'avec cet effort supplémentaire elle pourrait enrichir le champ des connaissances partagées. 
Sans sous-estimer le poids de ces contraintes socio-économiques, il nous semble qu'il y aurait profit pour la communauté à réfléchir aux moyens et conditions à mettre en place pour que les connaissances acquises dans le cadre des interventions entrent plus largement dans le champ public des connaissances ergonomiques.

Pour une part cela dépend des canaux de publication existants et de leur mode de fonctionnement. Les Actes des Congrès de la SELF sont l'un des principaux de ces canaux pour une bonne part des connaissances produites dans le cadre des interventions. Il s'agit là d'un vecteur à diffusion restreinte, pratiquement limitée aux inscrits aux congrès. La visibilité des textes ainsi publiés n'est donc pas des meilleures, et l'accès rétrospectif à ces textes pas des plus aisés. Mais on peut penser que cette question devrait désormais se présenter différemment grâce aux possibilités d'édition sous forme de CD-Rom; les ressources offertes par Internet pourraient fournir les moyens d'une meilleure pérennité de l'accès à ces textes.

D'autre part la lecture des Actes des Congrès de la SELF montre que, dans un certain nombre de cas le travail d'élaboration que suppose la publication des enseignements des interventions n'est pas toujours aussi poussé qu'il devrait l'être. On peut sur ce point se demander si cela ne reflète pas un niveau d'exigence inférieur à celui auquel seraient en mesure de répondre les contributeurs qui, faute d'y être incités par les procédures ordinaires d'acceptation des communications aux Congrès de la SELF, présentent parfois des communications qui paraissent rédigées à l'économie. Mais il existe d'autres canaux où les enseignements des interventions ergonomiques peuvent entrer dans le champ public : c'est notamment la politique explicitement affirmée par plusieurs revues en particulier parPistes, @ctivités ou encore Performances.

La publication des enseignements des interventions n'est pas l'étape ultime de l'intégration de ces enseignements dans le corpus des connaissances ergonomiques. Chaque publication n'est en effet qu'une pièce d'un vaste puzzle et ne prend toute sa valeur que dans la mesure où elle est raccordée aux autres. Ce travail d'articulation n'est pas nécessairement pris en charge par tels ou tels des acteurs de la communauté ergonomique. Il est en fait à la charge de quiconque en éprouve le besoin, y trouve intérêt et dispose des moyens et du temps nécessaire. Il peut ainsi demeurer en souffrance, ou bien rester une activité personnelle, privée, au seul bénéfice de celui qui l'accomplit ou se concrétiser par la rédaction d'un texte de synthèse dont la publication viendra enrichir, en le structurant, le corpus des connaissances ergonomiques. C'est là un travail dans lequel le métier d'intervenant ne permet guère de s'engager, sauf à bénéficier d'un soutien particulier. Un exemple intéressant à cet égard est la production de l'ouvrage collectif « Troubles musculo-squelettiques et travail » (Bourgeois et al., 2000) réalisé par le groupe de travail TMS du réseau ANACT composé de consultants et de chercheurs et qui a capitalisé les enseignements de nombreuses actions de prévention des TMS. C'est là une capitalisation exemplaire qui apparaît d'autant plus bienvenue qu'elle s'appuie sur des travaux dont un bon nombre n'apparaissent référencés que sous forme de documents non publiés.

La mise en circulation des savoirs produits par les interventions passe aussi par le vecteurs d'échanges oraux, aux traces éventuellement moins pérennes, dans le cadre de rencontres professionnelles (ayant pour objet l'état des connaissances relatives à telle ou telle catégorie d'activité de travail ou les problèmes posés par la pratique des interventions) qui débouchent souvent sur des publications, certaines plus élaborées que d'autres. La multiplication de ces rencontres dont s'accompagne la structuration croissante de la profession que relève Daniellou (2003a) laisse cependant subsister un écart certain entre le monde des ergonomes porteurs d'une visée d'intervention, appelant forcément une lecture particularisée des situations, et ceux qui sont porteurs d'une visée généralisante. Les liens tissés sont encore souvent assez lâches. Cela tient aux contraintes institutionnelles et socioéconomiques respectives des uns et des autres. Faire évoluer les points de vue des organismes de recherche et d'enseignement est une tâche lourde et de longue haleine, dans laquelle le rôle des universités a des chances d'être crucial. Si les praticiens ont, de par leur fonction même, une perspec- 
tive particularisante, celle-ci n'est pas incompatible avec le maintien, en arrière-plan de préoccupations généralisantes. Pour que celles-ci puissent rester vivantes peut-être faut-il qu'elles soient bien enracinées, plus qu'elles ne semblent l'être présentement, au stade de la formation initiale : celle-ci ne peut être simplement la présentation d'un savoir établi et définitif mais celle d'un savoir en permanente construction, constitué de résultats ayant un champ de validité à déterminer et de questions irrésolues. Des formations qui ne donneraient pas un poids suffisant à ce point de vue risqueraient d'entraîner, dans le long terme, une déperdition des potentialités de contribution des praticiens au progrès des connaissances ergonomiques.

\section{4.- Conclusion}

Champ de connaissances et pratique sociale, l'ergonomie conjugue différents types de savoirs qui se distinguent par leur nature, leur articulation avec d'autres champs disciplinaires, leurs modes et leurs conditions de production. Nous en avons proposé ici une catégorisation susceptible d'aider à clarifier les débats que ne cessent de susciter les rapports de la pratique et de la recherche, des praticiens et des chercheurs, et à en dépasser les trop fréquentes simplifications qui les rendent peu productifs. Les différentes composantes des connaissances ergonomiques ainsi précisées, il devrait être possible de dégager des pistes de réflexion plus ciblées dont l'exploration pourrait conduire à définir et engager des actions, concernant l'organisation et le fonctionnement de la profession, en vue d'accroître la densité des échanges entre les divers acteurs de la scène ergonomique, et de parvenir à un enrichissement réciproque des productions des uns et des autres.

Un objectif crucial est d'améliorer la circulation de l'information entre tous les acteurs contribuant, ou susceptibles de contribuer, dans des cadres différents, à la construction de modèles des activités de travail et des processus sociaux qui régissent l'organisation de celles-ci et leur transformation. En pratique, la complémentarité des différents types de savoirs distingués en première partie ne va pas sans difficultés, comme en témoigne notamment ce point de crispation que constitue l'idée rebattue d'une irrémédiable divergence des centres d'intérêt des praticiens et des chercheurs. Cette vision est, sur le plan des principes comme à l'aune de notre expérience, excessive et fausse. Chercheurs et praticiens ont beaucoup à se dire, et si la communication s'avère, en l'état actuel, difficile, chaotique et insatisfaisante, se retirer sur l'Aventin ne saurait être une solution satisfaisante.

Certes, certaines pesanteurs institutionnelles ne sont sans doute pas près de disparaitre : dans un cadre tel que le CNRS, en dépit des discours officiels et mis à part le domaine des techniques de l'information et de la communication, la valorisation de l'articulation de la recherche avec les pratiques retient peu l'intérêt. Mais il peut y avoir plus à espérer dans le cadre universitaire, tant pour l'irrigation des pratiques des intervenants par les avancées récentes de la recherche, que pour celle des recherches universitaires par les questions rencontrées sur le terrain par les intervenants. Dans cette perspective, on peut penser qu'il serait sans doute bon, qu'au niveau des cursus visant la formation à l'intervention, un accent plus marqué soit mis sur l'articulation des interventions avec le développement des recherches et sur les moyens et conditions de cette articulation. Il y aura d'autant mieux production de connaissances par les interventions que celles-ci seront articulées au départ avec les perspectives de recherche du moment dans le domaine. On mentionnera ici un travail canadien (Bourdhouxe \& Gratton, 2003) qui discute le problème du transfert et de l'utilisation des résultats d'études en milieu de travail et propose quelques principes d'analyse et des recommandations illustrées par un exemple.

Et au-delà de la formation initiale, le maintien de liens vivants entre les praticiens et les enseignants / chercheurs (ceux dont on a suivi les enseignements mais aussi les tenants d'autres points de vue) apparaît essentiel. La voie est pour partie déjà ouverte, soit à travers des réseaux tel, 
par exemple, le groupe Acting, ou des rencontres comme les journées de Bordeaux ou le Séminaire de Paris I.

Un point qui paraît particulièrement important et qui pourrait bénéficier d'interactions plus denses entre praticiens et chercheurs est celui de la capitalisation des connaissances produites dans le cadre des interventions. Celle-ci apparaît actuellement trop négligée au regard de ce qu'elle pourrait être. Certes les conditions institutionnelles et socio-économiques des interventions ergonomiques y sont sans doute pour beaucoup, mais il semble qu'un renforcement des échanges entre praticiens et enseignants / chercheurs, à travers les pistes évoquées précédemment, pourrait améliorer les choses. Un autre facteur qui devrait y aider est le développement de canaux de publications plus ouverts que ceux disponibles jusqu'ici aux apports des interventions, tels que Pistes et @ctivités, qui ont en commun cette ambition, chacun avec une politique éditoriale spécifique.

Ce renforcement des échanges devrait être aussi favorisé par les ressources de l'outil Internet dont l'usage reste à développer plus largement : qu'il s'agisse de l'accès aux sources de documentation ou à des réseaux et forums, comme par exemple le site d'échange d'expériences qui avait été ouvert par l'ANACT, pour la mise en place des 35 heures, à tous les intervenants agréés par l'Agence : il y a là des outils puissants qui devraient permettre une meilleure diffusion et valorisation des différents types de connaissances produites par tous les acteurs contribuant, chacun à leur place, à faire progresser les cadres d'analyse des activités de travail, de leurs conditions de réalisation et de leur transformation.

Cette esquisse de prospective, et les analyses précédentes qu'elle vient conclure, sont sans doute marquées par les places et les expériences qui sont les nôtres et, par là même, matière à discussion. Notre souhait est qu'elles soient prises pour telles et qu'elles contribuent à alimenter la réflexion collective.

\section{BIBLIOGRAPHIE}

Abisou, G. (1993). De l'étable à l'étal. Les conditions de travail dans la filière viande. Paris: ANACT.

Bisseret, A., Sebillotte, S., \& Falzon, P. (1999). Techniques pratiques pour l'étude des activités expertes. Toulouse: Octarès.

Bourdhouxe, M., \& Gratton, L. (2003). Transfert et utilisation des résultats en milieu de travail : le cas de la recherche sur les éboueurs au Québec. Pistes, 5, http://www.pistes.uqam.ca/v5n1/articles/v5n1a8.htm.

Bourgeois, F., Lemarchand, C., Hubault, F., Brun, C., Polin, A., \& Faucheux J. M. (2000). Troubles musculosquelettiques et travail, Paris: ANACT.

Daniellou, F. (1997). Evolutions de l'ergonomie francophone : théories, pratiques et théories de la pratique Actes du 32ème Congrès de la SELF, 37-56.

Daniellou, F. (1998). Une contribution au nécessaire recensement des Repères pour affronter les TMS. In TMS et évolution des conditions de travail. Paris: ANACT.

Daniellou, F. (2003a), Introduction. In C. Martin \& D. Baradat (Eds) (2003) Des pratiques en réflexion (pp. 722), Toulouse: Octarès.

Daniellou, F. (2003b). L'ergonome est-il un praticien? In C. Martin \& D. Baradat (Eds) (2003) Des pratiques en réflexion (pp. 23-32), Toulouse: Octarès.

Daniellou, F. (2003c). La construction de l'intervention ergonomique In C. Martin \& D. Baradat (Eds) (2003) Des pratiques en réflexion, (pp. 135-143), Toulouse: Octarès.

Falzon, P. (1997). La construction des connaissances en ergonomie : éléments d'épistémologie, Actes du 32ème Congrès de la SELF (pp. 641-654).

Falzon, P. (Ed.) (A paraître). Traité d'ergonomie. Paris: PUF.

Hella, F. (1983). Contribution de l'étude du champ visuel à la connaissance de l'espace de travail. Le Travail Humain, 46, 1, 11-32. 
Lamonde, F. (1997). Recherche, pratique et formation en ergonomie : vers le développement d'un programme culturel pour notre discipline. Actes du 32ème Congrès de la SELF (pp. 465-490).

Lamonde, F. (2000). L'intervention ergonomique. Un regard sur la pratique professionnelle. Toulouse: Octarès.

Leplat, J. (2002). De l'étude de cas à l'analyse de l'activité, Pistes, Vol 4, No 2. http://www.pistes.uqam.ca/ v4n2/articles/v4n2a8.htm

Leplat, J. (1997a). À propos des compétences incorporées In J. Leplat, Regards sur l'activité en situation de travail. Contribution à la psychologie ergonomique, (pp. 111-139). Paris: PUF.

Leplat, J. (1997b). Les lectures des ergonomes, In Recherche, Pratique, Formation en ergonomie, Actes du 32ème Congrès de la SELF (735-742).

Leplat, J. (1997c). La perspective clinique en psychologie ergonomique, In J. Leplat, Regards sur l'activité en situation de travail. Contribution à la psychologie ergonomique, (ch. 4). Paris : PUF.

Martin, C., \& Baradat D. (Eds). (2003). Des pratiques en réflexion, Toulouse: Octarès.

Mazeau, (2003). De l'ergonomie de problème à l'ergonomie de système : l'ergonome, un ingénieur comme les autres, In C. Martin \& D. (Eds), Des pratiques en réflexion (pp. 466-472), Toulouse: Octarès.

Prunier-Poulmaire, S. (1998). Les postes d'encaissement en hypermarchés et supermarchés : approche ergonomique du travail en vue de la conception des postes d'encaissement ». Publication de l'INRS, en partenariat avec la CNAM et PERIFEM, 36p.

Prunier-Poulmaire, S., \& Gadbois, C. (1998), Les effets combinés du travail posté : le cas des agents des Douanes, In Temps et travail, Actes du XXIIIème Congrès de la SELF.

Schön, D. A. (1983). The Reflexive Practitioner: How Professionals Think in Action. New York : Basic books.

Schön, D. A. (1987). Educating the Reflexive Practitioner. San Francisco: Jossey-Bass Publishers.

Vézina, N. (2001). La pratique de l'ergonomie face aux TMS : ouverture à l'interdisciplinarité, in Actes du Congrès SELF-ACE 2001.

Wisner, A. (1995). Réflexions sur l'ergonomie (1962-1995). Toulouse: Octarès. 